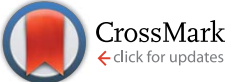

Cite this: J. Anal. At. Spectrom., 2014, 29, 1897

Received 6th June 2014

Accepted 15th July 2014

DOI: $10.1039 / c 4 j a 00183 d$

www.rsc.org/jaas

\section{Measurements of gamma-ray background spectra at spallation neutron source beamlines}

\author{
A. Miceli, ${ }^{a}$ G. Festa, ${ }^{\text {ab }}$ R. Senesi, ${ }^{\text {ab }}$ E. Perelli Cippo, ${ }^{c}$ L. Giacomelli, ${ }^{c}$ M. Tardocchi, ${ }^{c}$ \\ A. Scherillo, ${ }^{\text {be }}$ E. Schooneveld, ${ }^{e}$ C. Frost, ${ }^{e}$ G. Gorini ${ }^{d}$ and C. Andreani ${ }^{\text {ab }}$
}

The gamma-ray background spectra of VESUVIO and INES beamlines, at the ISIS spallation neutron source, were measured with an HPGe spectrometer. These measurements are intended to provide information on typical gamma-ray background spectra in spallation neutron source environments, with special regard to beamlines employing gamma-ray sensitive detectors. Sources and creation processes of more than 60 gamma-ray lines in the energy range $80 \mathrm{keV}-12 \mathrm{MeV}$ were identified by using specifically developed data analysis software.

\section{Introduction}

Gamma-ray background is often the main background source in neutron beamlines at new generation spallation facilities. The gamma-ray components are produced within the target/ reflector/moderator assemblies, in the beamline components, such as guides/shielding, in the sample area and in the surrounding enclosure. Characterization of the gamma background on a neutron spectrometer is of paramount importance to determine its best operational conditions. This is particularly relevant for beamlines employing gamma-sensitive detectors, ${ }^{1,2,5-7}$ for example CCD assemblies in new generation imaging beamlines or spectrometers equipped with solid state scintillators. In this respect a typical figure of merit for neutron scintillators is represented by the number of gamma photons required to give the same light output as one neutron, which varies from $10^{2}$ for $0.6 \mathrm{MeV}$ gamma-rays to $10^{1}$ for $1 \mathrm{MeV}$ gamma-rays. Threshold discrimination techniques become less effective for high-count rate neutron detectors envisaged for the new generation of neutron instruments, with special regard to the use of ${ }^{3} \mathrm{He}$-free detector materials. In particular this kind of detector is more sensitive to gamma background since the pulse height spectral signal associated with gamma background is less easily separated from the neutron pulse height spectrum through low level discrimination methods.

At spallation sources or reactor facilities, where the incident neutron beam at the sample position is mainly moderated, gamma-rays are primarily produced by radiative neutron

${ }^{a}$ Department of Physics and NAST Centre, University of Rome Tor Vergata, Rome, Italy. E-mail: giulia.festa@roma2.infn.it

${ }^{b}$ CNR-IPCF Sezione di Messina, Messina, Italy

'Istituto di Fisica del Plasma, Associazione EURATOM-ENA/CNR, Milano, Italy

${ }^{d}$ Department of Physics, University of Milano-Bicocca, Milan, Italy

${ }^{e}$ ISIS Facility, STFC, Chilton, Didcot, United Kingdom capture and activation. Some gamma-rays are produced in inelastic neutron scattering from under moderated neutrons. In the literature, studies on gamma-ray background at neutron experimental beamlines are very limited and in particular at spallation neutron sources do not cover a large energy range..$^{\mathbf{8 - 1 1}}$ In this paper, a thorough study of the gamma-ray signal on the VESUVIO and INES beamlines at ISIS in the energy window $80 \mathrm{keV}-12 \mathrm{MeV}$ is illustrated.

\section{Experimental set-up}

The VESUVIO spectrometer ${ }^{12}$ is an inelastic neutron scattering spectrometer for high momentum and energy transfers, for the measurement of atomic momentum distributions in condensed matter systems. Schematic layout of the beamline is reported in Fig. 2. VESUVIO makes use of epithermal neutrons in excess of 1 $\mathrm{eV}$ and the time of flight technique to carry out experiments in the Deep Inelastic Neutron Scattering (DINS) regime. ${ }^{13}$ The instrument is installed on a neutron beamline viewing a room temperature $\mathrm{H}_{2} \mathrm{O}$ moderator. The neutron beam travels for $11.055 \mathrm{~m}$ from moderator to sample, located inside an $\mathrm{Al}$ sample tank, passing through a series of collimators made of stainless steel and boron carbide. Most experiments are designed such that neutron scattering from samples is kept lower than $20 \%$. Thus a large part of the incident neutron beam is transmitted through the sample, hits the beam dump and produces an intense gamma ray emission. ${ }^{\mathbf{1 1}}$ The neutron source spectrum on the VESUVIO beamline at a measurement position of approximately $12 \mathrm{~m}$ from the moderator i.e. close to the measurement position of the present experiment is shown in Fig. 1 and reported in ref. 3 .

The beam dump, $5 \mathrm{~m}$ from the sample position, is mostly composed of hydrogen, boron, and iron. ${ }^{11}$ Further neutrons scattered by the sample or by the beam dump hit the 


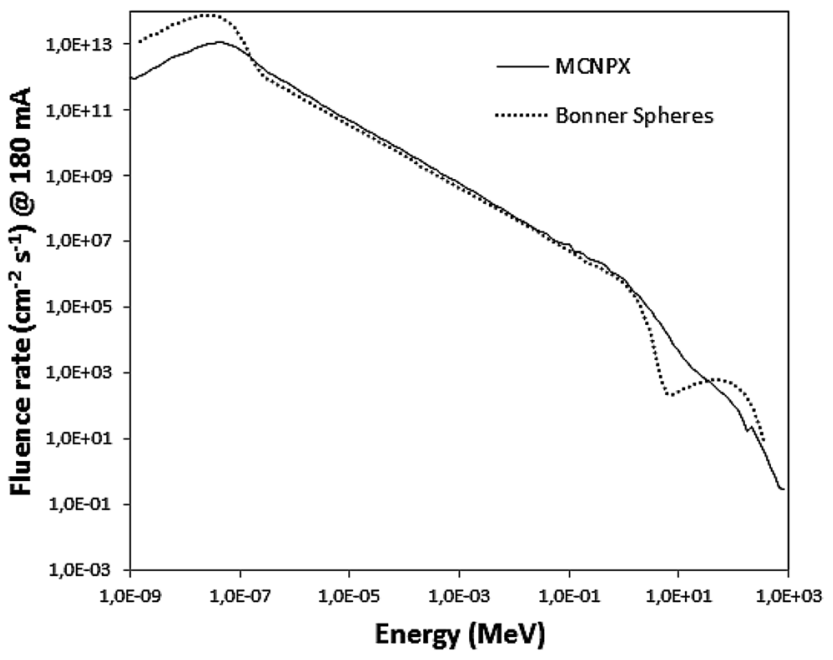

Fig. 1 Simulated and measured neutron spectra at the VESUVIO beamline. $^{3}$

-

blockhouse walls, sample tanks, pipes, and other blockhouse components producing gammas.

The INES beamline ${ }^{\mathbf{1 4}}$ is a general purpose diffractometer and is mainly devoted to materials characterization (structure refinement and phase analysis), cultural heritage studies and equipment tests. Schematic layout of the beamline is reported in Fig. 2. INES is installed on a guide viewing the beam transmitted to the primary TOSCA spectrometer, which uses a room temperature $\mathrm{H}_{2} \mathrm{O}$ moderator with the sample position located at $22.804 \mathrm{~m}$ from the moderator. Thus the neutron flux is about 4 times less intense than on the VESUVIO beamline. On INES the beam dump is closer to the sample position at about $1.3 \mathrm{~m}$. A list of chemical elements present in the components of the beamline layout according to the beamline engineering drawings is reported in Table 1.
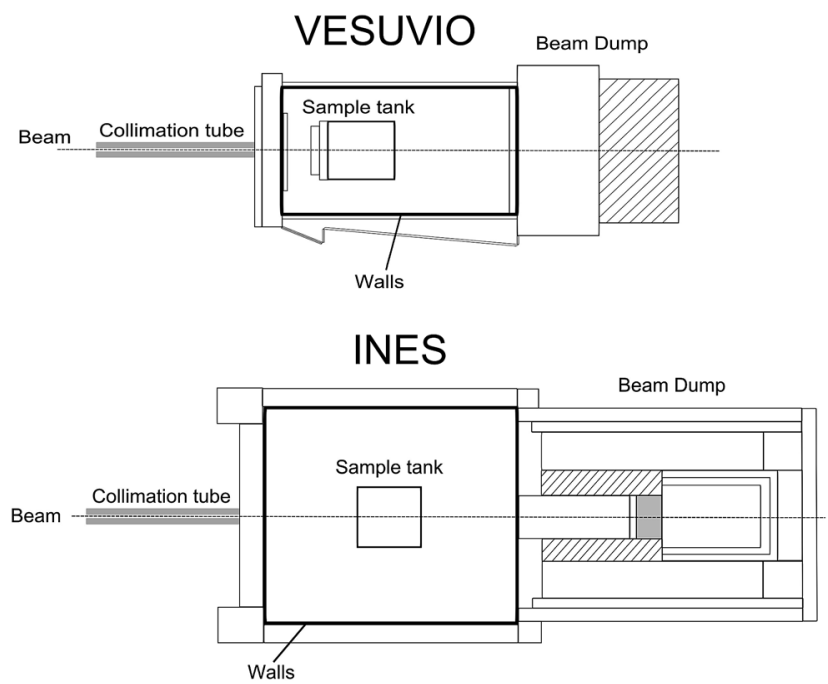

Fig. 2 Schematic layouts of the VESUVIO and INES beamlines.
Table 1 Chemical elements and components evinced from beamline drawings

\begin{tabular}{ll}
$\begin{array}{l}\text { Chemical } \\
\text { elements }\end{array}$ & Components \\
\hline $\mathrm{H}$ & Walls, beam dump, moderator $\left(\mathrm{H}_{2} \mathrm{O}\right)$ \\
$\mathrm{Li}$ & Neutron monitor, backscattering detectors (VESUVIO) \\
$\mathrm{B}$ & Walls, beam dump, collimator $\left(\mathrm{B}_{4} \mathrm{C}\right)$ \\
$\mathrm{C}$ & Collimator $\left(\mathrm{B}_{4} \mathrm{C}\right)$ \\
$\mathrm{O}$ & Moderator $\left(\mathrm{H}_{2} \mathrm{O}\right)$, air, walls, beam dump \\
$\mathrm{N}$ & Air, resins $\left(\mathrm{B}_{4} \mathrm{C}\right.$ binder) \\
$\mathrm{Al}$ & Sample tank, collimator \\
$\mathrm{Fe}$ & Beam dump, flange, pipe, collimator (stainless steel \\
& grade 316L) \\
$\mathrm{Ge}$ & HPGe gamma-ray detector \\
$\mathrm{Cd}$ & Foils on VESUVIO detectors \\
$\mathrm{Sb}$ & Lead shielding \\
$\mathrm{Pb}$ & Lead shielding, upstream collimator \\
$\mathrm{Cr}$ & Beam dump, flange, pipe, collimator (stainless steel \\
$\mathrm{Mn}$ & grade 316L) \\
$\mathrm{Me}$ & Beam dump, flange, pipe, collimator (stainless steel \\
$\mathrm{Ti}$ & grade 316L) \\
$\mathrm{V}$ & Beam pipe (aluminium alloys) \\
$\mathrm{Na}$ & Collimator (silver steel) \\
& Collimator (borax)
\end{tabular}

Measurements of the gamma-ray spectra on the VESUVIO and INES beamlines were performed using an ORTEC-GMX ${ }^{15}$ HPGe spectrometer. The detector was covered with borated plastic to reduce the number of interactions of scattered neutrons with HPGe. The HPGe spectrometer was placed at a scattering angle of 45 degrees vertically aligned with the center of the neutron beam. On VESUVIO, due to geometrical constrains the spectrometer was placed $1 \mathrm{~m}$ behind the sample tank. The HPGe signal was fed to an ORTEC-DSPEC50 (ref. 15) digital spectrometer which provided low voltage to the preamplifier and high voltage to the HPGe. The pulse height spectra were acquired using the ORTEC MAESTRO $\mathrm{MCA}^{15}$ acquisition software. Shaping parameters were $12 \mu$ s for the rise time and fall time, $0.8 \mu$ s for the flat top. The estimated dead time was $37.6 \mu \mathrm{s}$. The internal lower level discriminator was set to $78 \mathrm{keV}$ on VESUVIO and $153 \mathrm{keV}$ on INES in order to limit the dead time. Data were acquired for $1 \mathrm{~h}$ and $40 \mathrm{~min}$.

\section{Data analysis}

Peak detection and element identification software based on ROOT $^{16,17}$ and C++ was developed. The software (i) converts the MAESTRO-ORTEC ${ }^{15}$ data format into a ROOT format, (ii) outputs peak locations, (iii) lists the possible elements for each peak, and (iv) assigns the most probable element to each peak relative to a published prompt gamma-ray database.

\subsection{Peak detection}

The aim of the peak detection algorithm was to find the centroid of the peaks in the gamma-ray spectrum. This allowed us to identify the chemical elements. We used a ROOT advanced spectra processing function TSpectrum to characterize and 
Table 2 VESUVIO gamma-ray background lines. Peaks assigned to the chemical elements of Table 1 are in bold ${ }^{c}$

\begin{tabular}{|c|c|c|c|}
\hline Energy (keV) & Element & Process & Notes \\
\hline $122.2 \pm 1.6$ & $\mathrm{~V}$ & $\mathrm{C}$ & \\
\hline $140.6 \pm 1.4$ & ${ }^{75 \mathrm{~m}} \mathrm{Ge}$ & A & $\begin{array}{l}139.96 \mathrm{keV}^{75 \mathrm{~m}} \mathrm{Ge}^{a} \\
139.68 \mathrm{keV}^{75 \mathrm{~m}} \mathrm{Ge}^{b}\end{array}$ \\
\hline $162.8 \pm 1.6^{*}$ & In, ${ }^{74} \mathrm{Ge}$ & A, C & $162.54 \mathrm{keV}^{74} \mathrm{Ge}^{a}$ \\
\hline $199.1 \pm 1.5^{*}$ & ${ }^{71} \mathrm{~m} G e$ & A & $\begin{array}{l}198.53 \mathrm{keV}^{71 \mathrm{~m}} \mathrm{Ge}^{a} \\
198.39 \mathrm{keV}^{71 \mathrm{~m}} \mathrm{Ge}^{b}\end{array}$ \\
\hline $212.1 \pm 1.7$ & Mn & $\mathrm{C}$ & \\
\hline $353.0 \pm 1.4^{*}$ & $\mathrm{Fe}$ & $\mathrm{C}$ & \\
\hline $417.3 \pm 2.0^{*}$ & ${ }^{116 \mathrm{~m}} \mathrm{In}$ & A & $416.86^{116 \mathrm{~m}} \mathrm{In}^{b}$ \\
\hline $477.3 \pm 3.8^{*}$ & ${ }^{10} \mathrm{~B}$ & $(n, \alpha)$ & $477.90 \mathrm{keV}^{10} \mathrm{~B}^{a}$ \\
\hline $511.4 \pm 2.1 *$ & & AN & $\begin{array}{l}511.06 \mathrm{keV}^{a} \\
511.00^{b}\end{array}$ \\
\hline $560.0 \pm 3.3$ & $\mathrm{Cd}$ & $\mathrm{C}$ & \\
\hline $596.3 \pm 1.4^{*}$ & ${ }^{74} \mathrm{Ge}$ & $\mathrm{C}, \mathrm{IS}$ & $\begin{array}{l}595.86 \mathrm{keV} \text { C, IS }{ }^{74} \mathrm{Ge}^{a} \\
595.85 \mathrm{keV} \text { IS }{ }^{74} \mathrm{Ge}^{b}\end{array}$ \\
\hline $609.8 \pm 1.2^{*}$ & ${ }^{74} \mathrm{Ge}$ & IS & $608.3 \mathrm{keV}^{74} \mathrm{Ge}^{a}$ \\
\hline $691.9 \pm 2.1 *$ & ${ }^{72} \mathrm{Ge},{ }^{57} \mathrm{Fe}$ & IS, C & $\begin{array}{l}693.05 \mathrm{keV}^{72} \mathrm{Ge}^{a},{ }^{57} \mathrm{Fe}^{a} \\
689.6 \mathrm{keV}^{72} \mathrm{Ge}^{b}\end{array}$ \\
\hline $708.0 \pm 1.3^{*}$ & ${ }^{71} \mathrm{Ge}$ & $\mathrm{C}$ & $708.4 \mathrm{keV}^{71} \mathrm{Ge}^{a}$ \\
\hline $748.8 \pm 1.9 *$ & $\mathrm{Cr}$ & $\mathrm{C}$ & \\
\hline $787.8 \pm 2.0^{*}$ & $\mathrm{Cl}$ & $\mathrm{C}$ & \\
\hline $818.9 \pm 1.4^{*}$ & In, Sn & $\mathrm{A}, \mathrm{C}$ & \\
\hline $834.9 \pm 1.4^{*}$ & $\mathrm{Cr},{ }^{28} \mathrm{Al}$ & $\mathrm{C}$ & $\begin{array}{l}831.8 \mathrm{keV}^{28} \mathrm{Al}^{a} \\
832.01 \mathrm{keV}^{211} \mathrm{~Pb}^{b}\end{array}$ \\
\hline $846.9 \pm 1.6^{*}$ & $\mathrm{Mn},{ }^{56} \mathrm{Fe}$ & A, IS & $\begin{array}{l}846.85 \mathrm{keV}^{56} \mathrm{Fe}^{a} \\
846.77 \mathrm{keV}^{56} \mathrm{Fe}^{b}\end{array}$ \\
\hline $871.3 \pm 0.2$ & ${ }^{57} \mathrm{Fe},{ }^{17} \mathrm{O}$ & $\mathrm{C}$ & $870.75 \mathrm{keV}^{57} \mathrm{Fe}^{a},{ }^{17} \mathrm{O}^{a}$ \\
\hline $898.4 \pm 1.5^{*}$ & ${ }^{207} \mathrm{~Pb},{ }^{57} \mathrm{Fe}$ & IS, C & $896.56 \mathrm{keV} \mathrm{IS}{ }^{207} \mathrm{~Pb}^{a}, \mathrm{C}^{57} \mathrm{Fe}^{a}$ \\
\hline $971.6 \pm 2.6$ & $\mathrm{Sn}$ & $\mathrm{C}$ & \\
\hline $983.3 \pm 2.1^{*}$ & $\mathrm{Al}$ & $\mathrm{C}$ & \\
\hline $1097.2 \pm 1.6^{*}$ & ${ }^{116}$ In & A & $1097.3 \mathrm{keV}^{116} \operatorname{In}^{b}$ \\
\hline $1164.5 \pm 1.7^{*}$ & $\mathrm{Cl}$ & $\mathrm{C}$ & \\
\hline $1172.3 \pm 1.8^{*}$ & $\mathrm{Sn},{ }^{60} \mathrm{Co}$ & $\mathrm{C}, \mathrm{a}$ & $\begin{array}{l}1173.24 \mathrm{keV} \mathrm{A}^{60} \mathrm{Co}^{a} \\
1173.23 \mathrm{keV} \mathrm{A}^{60} \mathrm{Co}^{b}\end{array}$ \\
\hline $1204.8 \pm 0.4^{*}$ & $\mathrm{Ge}$ & $\mathrm{C}$ & \\
\hline $1293.2 \pm 1.7^{*}$ & ${ }^{116} \mathrm{In}, \mathrm{Sn}$ & $\mathrm{A}, \mathrm{C}$ & $1293.54 \mathrm{keV}{ }^{116} \operatorname{In}^{b}$ \\
\hline $1367.4 \pm 1.6$ & $\mathrm{Na}$ & A & \\
\hline $1433.0 \pm 1.8^{*}$ & $\mathrm{~V}$ & A & \\
\hline $1507.2 \pm 1.8^{*}$ & In & A & \\
\hline $1601.0 \pm 2.0^{*}$ & $\mathrm{Cl}$ & $\mathrm{C}$ & \\
\hline $1612.5 \pm 1.7^{*}$ & $\mathrm{Fe}$ & $\mathrm{C}$ & \\
\hline $1725.3 \pm 1.8^{*}$ & ${ }^{57} \mathrm{Fe}$ & $\mathrm{C}$ & $1725.5 \mathrm{keV}{ }^{57} \mathrm{Fe}^{a}$ \\
\hline $1752.0 \pm 2.3^{*}$ & In & A & \\
\hline $1778.9 \pm 1.8^{*}$ & ${ }^{28} \mathrm{Al}$ & A & $1778.92 \mathrm{keV}^{28} \mathrm{Al}^{a}$ \\
\hline $1810.7 \pm 1.7^{*}$ & $\mathrm{Mn}$ & A & \\
\hline $1885.1 \pm 2.3$ & ${ }^{15} \mathrm{~N}$ & $\mathrm{C}$ & $1884.72 \mathrm{keV}{ }^{15} \mathrm{~N}^{a}$ \\
\hline $1950.9 \pm 1.8^{*}$ & $\mathrm{Cl}$ & $\mathrm{C}$ & \\
\hline $2112.9 \pm 1.8^{*}$ & $\mathrm{Mn}, \mathrm{In}, \mathrm{Sn}$ & $\mathrm{A}, \mathrm{A}, \mathrm{C}$ & \\
\hline $2223.6 \pm 1.8^{*}$ & ${ }^{2} \mathrm{H}$ & $\mathrm{C}$ & $\begin{array}{l}2223.25 \mathrm{keV}^{2} \mathrm{H}^{a} \\
2224.57 \mathrm{keV}^{2} \mathrm{H}^{b}\end{array}$ \\
\hline $2240.7 \pm 4.4$ & $\mathrm{Cr}$ & $\mathrm{C}$ & \\
\hline $2615.2 \pm 1.9$ & ${ }^{208} \mathrm{~Pb}$ & IS & $\begin{array}{l}2614.58 \mathrm{keV}^{208} \mathrm{~Pb}^{a} \\
2614.51 \mathrm{keV}^{208} \mathrm{~Pb}^{b}\end{array}$ \\
\hline $2754.4 \pm 1.8^{*}$ & $\mathrm{Na}$ & A & \\
\hline $2959.5 \pm 2.4^{*}$ & $\mathrm{Mn},{ }^{28} \mathrm{Al}$ & $\mathrm{C}$ & $2960.01 \mathrm{keV}^{28} \mathrm{Al}^{a}$ \\
\hline $3412.7 \pm 2.6^{*}$ & ${ }^{57} \mathrm{Fe}$ & $\mathrm{C}$ & $3413.1 \mathrm{keV}^{57} \mathrm{Fe}^{a}$ \\
\hline $3436.6 \pm 2.5^{*}$ & ${ }^{57} \mathrm{Fe}$ & $\mathrm{C}$ & $3436.0 \mathrm{keV}^{57} \mathrm{Fe}^{a}$ \\
\hline $4132.6 \pm 1.5^{*}$ & ${ }^{28} \mathrm{Al}$ & $\mathrm{C}$ & $4133.1 \mathrm{keV}^{28} \mathrm{Al}^{a}$ \\
\hline $4217.8 \pm 2.8^{*}$ & ${ }^{57} \mathrm{Fe}$ & $\mathrm{C}$ & $4218.6 \mathrm{keV}^{57} \mathrm{Fe}^{a}$ \\
\hline $4259.3 \pm 1.4^{*}$ & ${ }^{28} \mathrm{Al}$ & $\mathrm{C}$ & $4259.46 \mathrm{keV}^{28} \mathrm{Al}^{a}$ \\
\hline $5410.0 \pm 3.1^{*}$ & ${ }^{28} \mathrm{Al}$ & $\mathrm{C}$ & $5410.6 \mathrm{keV}^{28} \mathrm{Al}^{a}$ \\
\hline $5920.3 \pm 2.6^{*}$ & ${ }^{57} \mathrm{Fe}$ & $\mathrm{C}$ & $5920.41 \mathrm{keV}^{57} \mathrm{Fe}^{a}$ \\
\hline
\end{tabular}

Table 2 (Contd.)

\begin{tabular}{llll}
\hline Energy $(\mathrm{keV})$ & Element & Process & Notes \\
\hline $\mathbf{6 0 1 8 . 8} \pm \mathbf{2 . 5}$ & ${ }^{57} \mathrm{Fe}$ & $\mathrm{C}$ & $6017.75 \mathrm{keV}^{57} \mathrm{Fe}^{a}$ \\
$\mathbf{6 1 1 1 . 0} \pm 3.8^{*}$ & ${ }^{36} \mathrm{Cl}$ & $\mathrm{C}$ & $6111.6 \mathrm{keV}^{36} \mathrm{Cl}^{a}$ \\
$\mathbf{6 7 6 8 . 5} \pm \mathbf{2 . 5}$ & ${ }^{7} \mathrm{Li}$ & $\mathrm{C}$ & $6769.4 \mathrm{keV}^{7} \mathrm{Li}^{a}$ \\
$\mathbf{7 1 2 1 . 0} \pm \mathbf{3 . 3}$ & ${ }^{57} \mathrm{Fe}$ & $\mathrm{C}$ & $7120.3 \mathrm{keV}^{57} \mathrm{Fe}^{a}$ \\
$\mathbf{7 1 3 4 . 9} \pm \mathbf{3 . 2}$ & ${ }^{57} \mathrm{Fe}$ & $\mathrm{C}$ & $7134.0 \mathrm{keV}^{57} \mathrm{Fe}^{a}$ \\
$\mathbf{7 2 1 3 . 5} \pm \mathbf{3 . 4}$ & ${ }^{28} \mathrm{Al}$ & $\mathrm{C}$ & $7212.8 \mathrm{keV}^{28} \mathrm{Al}^{a}$ \\
$\mathbf{7 2 7 9 . 1} \pm \mathbf{3 . 4}$ & ${ }^{57} \mathrm{Fe}$ & $\mathrm{C}$ & $7278.3 \mathrm{keV}^{57} \mathrm{Fe}^{a}$ \\
$\mathbf{7 6 3 2 . 1} \pm \mathbf{3 . 1}$ & ${ }^{57} \mathrm{Fe}$ & $\mathrm{C}$ & $7631.0 \mathrm{keV}^{57} \mathrm{Fe}^{a}$ \\
$\mathbf{7 6 4 6 . 2} \pm \mathbf{3 . 2}$ & ${ }^{57} \mathrm{Fe}$ & $\mathrm{C}$ & $7645.6 \mathrm{keV}^{57} \mathrm{Fe}^{a}$ \\
$\mathbf{7 7 2 5 . 2} \pm \mathbf{3 . 0}$ & ${ }^{28} \mathrm{Al}$ & $\mathrm{C}$ & $7723.85 \mathrm{keV}^{28} \mathrm{Al}^{a}$ \\
$\mathbf{8 8 8 4 . 0} \pm \mathbf{2 . 0}$ & ${ }^{\mathrm{Cr}}$ & $\mathrm{C}$ & \\
$\mathbf{9 2 9 8 . 7} \pm \mathbf{2 . 4}$ & ${ }^{55} \mathrm{Fe}$ & $\mathrm{C}$ & $9295.8 \mathrm{keV}^{55} \mathrm{Fe}^{a}$ \\
$\mathbf{9 7 1 8 . 9} \pm \mathbf{4 . 8}$ & $\mathrm{Cr}$ & $\mathrm{C}$ & \\
$\mathbf{1 0 8 3 0 . 1} \pm \mathbf{3 . 9}$ & ${ }^{15} \mathrm{~N}$ & $\mathrm{C}$ & $10829.1 \mathrm{keV}^{15} \mathrm{~N}^{a}$
\end{tabular}

${ }^{a}$ T. Belgya and Z. Revay and G. L. Molnar. ${ }^{10}{ }^{b}$ G. Gilmore and J. D. Hemingway. ${ }^{23}{ }^{c} \mathrm{~A}=$ activation, $\mathrm{AN}=$ annihilation, $\mathrm{C}=$ neutron capture, IS = inelastic scattering.

subtract the Compton continuum and to find the peaks. The full reference is given in ref. 18-20. Peaks with amplitude less than threshold (0.015) times the highest peak were discarded. In addition, to eliminate false peaks we used a three-step rejection method. First, we rejected peaks in the noise of the continuous spectrum by using the following equation: $\left(y_{\mathrm{p}}-\sqrt{y_{\mathrm{p}}}\right)<y_{\mathrm{b}}$ where $y_{\mathrm{p}}$ is the peak height and $y_{\mathrm{b}}$ is the peak background. Secondly, we rejected peaks narrower than 2 bins: $y_{\mathrm{p}}<y_{\mathrm{p}-2}$ where $y_{\mathrm{p}-2}$ is the number of counts in the bin position $p-2$. Approximately hundred peaks were found. Each peak was fitted with a gaussian:

$$
\frac{1}{\sigma \sqrt{2 \pi}} \mathrm{e}^{\frac{-(x-\mu)^{2}}{2 \sigma^{2}}}
$$

where $\mu$ is the centroid and $\sigma$ is the standard deviation. The lists of centroids and their standard deviation, derived from the fitting procedure, are reported in Tables 2 and 3.

\subsection{Elemental identification}

A prompt gamma activation analysis database published by Molnar $^{21}$ and the International Atomic Energy Agency ${ }^{22}$ was used to match peaks with chemical elements. The database also included delay gamma-rays. The database was converted in a ROOT format and values for energy $E_{\mathrm{DB}}$, partial gamma-ray production cross-section $\sigma_{\gamma}$, atomic number $Z$, mass number $A$, and type (prompt or delay) were recorded and stored. Only gamma-rays with $\sigma_{\gamma}$ above 0.01 barns were considered. The ROOT database was then sorted in descending order (i.e. most probably gamma-rays first). For each peak, all the gamma-rays in the database with energy $E_{\mathrm{p}}-2 \mathrm{keV}<E_{\mathrm{DB}}<E_{\mathrm{p}}+2 \mathrm{keV}$ were stored and the most probable element, i.e. the element with the highest weighted probability, was assigned to the peak. The weight assigned to each peak was 1 for the chemical elements evinced from the beamline drawings $(\mathrm{H}, \mathrm{Li}, \mathrm{B}, \mathrm{C}, \mathrm{O}, \mathrm{N}, \mathrm{Al}, \mathrm{Fe}$, $\mathrm{Ge}, \mathrm{Cd}, \mathrm{Sb}$, and $\mathrm{Pb}$ ). An additional check was done on the 
Table 3 INES gamma-ray background lines. Peaks assigned to the chemical elements of Table 1 are in bold ${ }^{c, d}$

\begin{tabular}{|c|c|c|c|}
\hline Energy (keV) & Element & Process & Notes \\
\hline $163.5 \pm 1.7^{*}$ & In, ${ }^{74} \mathrm{Ge}$ & $\mathrm{A}, \mathrm{C}$ & $162.54 \mathrm{keV}^{74} \mathrm{Ge}^{a}$ \\
\hline $199.5 \pm 0^{*}{ }^{*}$ & ${ }^{71 \mathrm{~m}} \mathrm{Ge}$ & A & $\begin{array}{l}198.53 \mathrm{keV}^{71 \mathrm{~m}} \mathrm{Ge}^{a} \\
198.39 \mathrm{keV}^{71 \mathrm{~m}} \mathrm{Ge}^{b}\end{array}$ \\
\hline $353.3 \pm 0.9^{*}$ & $\mathrm{Fe}$ & $\mathrm{C}$ & \\
\hline $417.8 \pm 1.0^{*}$ & ${ }^{116 m}$ In & A & $416.86 \mathrm{keV}^{116 \mathrm{~m}} \mathrm{In}^{b}$ \\
\hline $477.9 \pm 4.1^{*}$ & ${ }^{10} \mathrm{~B}$ & $(n, \alpha)$ & $477.90 \mathrm{keV}^{10} \mathrm{~B}^{a}$ \\
\hline $500.8 \pm 1.1$ & ${ }^{71} \mathrm{Ge}$ & $\mathrm{C}$ & $500.09 \mathrm{keV}^{71} \mathrm{Ge}^{a}$ \\
\hline $511.7 \pm 1.5^{*}$ & & AN & $\begin{array}{l}511.06 \mathrm{keV}^{a} \\
511.00 \mathrm{keV}^{b}\end{array}$ \\
\hline $596.6 \pm 1.0^{*}$ & ${ }^{74} \mathrm{Ge}$ & C, IS & $\begin{array}{l}595.86 \mathrm{keV}^{74} \mathrm{Ge}^{a} \\
595.85 \mathrm{keV} \text { IS }{ }^{74} \mathrm{Ge}^{b}\end{array}$ \\
\hline $609.4 \pm 0.9^{*}$ & ${ }^{74} \mathrm{Ge}$ & IS & $608.3 \mathrm{keV}^{74} \mathrm{Ge}^{a}$ \\
\hline $692.5 \pm 1.0^{*}$ & ${ }^{72} \mathrm{Ge},{ }^{57} \mathrm{Fe}$ & IS, C & $\begin{array}{l}693.05 \mathrm{keV}^{72} \mathrm{Ge}^{a},{ }^{57} \mathrm{Fe}^{a} \\
689.6 \mathrm{keV}^{72} \mathrm{Ge}^{b}\end{array}$ \\
\hline $708.7 \pm 1.0^{*}$ & ${ }^{71} \mathrm{Ge}$ & $\mathrm{C}$ & $708.4 \mathrm{keV}^{71} \mathrm{Ge}^{a}$ \\
\hline $749.0 \pm 1.1^{*}$ & $\mathrm{Cr}$ & $\mathrm{C}$ & \\
\hline $788.0 \pm 1.8^{*}$ & $\mathrm{Cl}$ & $\mathrm{C}$ & \\
\hline $819.2 \pm 0.9^{*}$ & In, Sn & $\mathrm{A}, \mathrm{C}$ & \\
\hline $833.6 \pm 2.1^{*}$ & $\mathrm{Cr},{ }^{28} \mathrm{Al}$ & $\mathrm{C}$ & $\begin{array}{l}831.8 \mathrm{keV}^{28} \mathrm{Al}^{a} \\
832.01 \mathrm{keV}^{211} \mathrm{~Pb}^{b}\end{array}$ \\
\hline $847.1 \pm 1.0^{*}$ & $\mathrm{Mn},{ }^{56} \mathrm{Fe}$ & A, IS & $\begin{array}{l}846.85 \mathrm{keV}^{56} \mathrm{Fe}^{a} \\
846.77 \mathrm{keV}^{56} \mathrm{Fe}^{b}\end{array}$ \\
\hline $898.8 \pm 0.9^{*}$ & ${ }^{207} \mathrm{~Pb},{ }^{57} \mathrm{Fe}$ & IS, C & $896.56 \mathrm{keV}$ IS ${ }^{207} \mathrm{~Pb}^{a}, \mathrm{C}^{57} \mathrm{Fe}^{a}$ \\
\hline $983.3 \pm 1.2^{*}$ & $\mathrm{Al}$ & $\mathrm{C}$ & \\
\hline $1097.3 \pm 1.1^{*}$ & ${ }^{116}$ In & A & $1097.3 \mathrm{keV}^{116} \operatorname{In}^{b}$ \\
\hline $1164.8 \pm 1.1^{*}$ & $\mathrm{Cl}$ & $\mathrm{C}$ & \\
\hline $1172 *$ & $\mathrm{Sn},{ }^{60} \mathrm{Co}$ & $\mathrm{C}, \mathrm{A}$ & $\begin{array}{l}\text { 1173.24 keV A }{ }^{60} \mathrm{Co}^{a} \\
1173.23 \mathrm{keV} \mathrm{A}{ }^{60} \mathrm{Co}^{b}\end{array}$ \\
\hline $1203.6 \pm 2.5^{*}$ & $\mathrm{Ge}$ & $\mathrm{C}$ & \\
\hline $1260.3 \pm 1.6$ & ${ }^{13} \mathrm{C}, \mathrm{Fe}$ & $\mathrm{C}$ & $1261.5 \mathrm{keV}^{13} \mathrm{C}^{a}, \mathrm{Fe}^{a}$ \\
\hline $1293.5 \pm 1.1^{*}$ & ${ }^{116} \mathrm{In}, \mathrm{Sn}$ & $\mathrm{A}, \mathrm{C}$ & $1293.54 \mathrm{keV}^{116} \mathrm{In}^{b}$ \\
\hline $1379.9 \pm 1.7$ & $\mathrm{Ti}$ & $\mathrm{C}$ & \\
\hline $1432.0 \pm 1.4^{*}$ & V & A & \\
\hline $1507.7 \pm 1.4^{*}$ & In & A & \\
\hline $1600.2 \pm 1.3^{*}$ & $\mathrm{Cl}$ & $\mathrm{C}$ & \\
\hline $1612.9 \pm 1.2^{*}$ & $\mathrm{Fe}$ & $\mathrm{C}$ & \\
\hline $1623.0 \pm 1.3$ & $\mathrm{Al}$ & $\mathrm{C}$ & \\
\hline $1725.4 \pm 1.4^{*}$ & ${ }^{57} \mathrm{Fe}$ & $\mathrm{C}$ & $1725.5 \mathrm{keV}^{57} \mathrm{Fe}^{a}$ \\
\hline $1752.5 \pm 1.4^{*}$ & In & A & \\
\hline $1779.0 \pm 1.2^{*}$ & ${ }^{28} \mathrm{Al}$ & A & $1778.92 \mathrm{keV}^{28} \mathrm{Al}^{a}$ \\
\hline $1810.7 \pm 1.2^{*}$ & Mn & A & \\
\hline $1951.2 \pm 1.0^{*}$ & $\mathrm{Cl}$ & $\mathrm{C}$ & \\
\hline $1959.6 \pm 1.3$ & $\mathrm{Cl}$ & $\mathrm{C}$ & \\
\hline $2112.6 \pm 1.3^{*}$ & $\mathrm{Mn}, \mathrm{In}, \mathrm{Sn}$ & $\mathrm{A}, \mathrm{A}, \mathrm{C}$ & \\
\hline $2223.4 \pm 1.3^{*}$ & ${ }^{2} \mathrm{H}$ & $\mathrm{C}$ & $\begin{array}{l}2223.25 \mathrm{keV}^{2} \mathrm{H}^{a} \\
2224.57 \mathrm{keV}^{2} \mathrm{H}^{b}\end{array}$ \\
\hline $2282.8 \pm 1.2$ & ${ }^{28} \mathrm{Al}$ & $\mathrm{C}$ & $2282.7 \mathrm{keV}^{28} \mathrm{Al}^{a}$ \\
\hline $2590.6 \pm 1.2$ & ${ }^{28} \mathrm{Al}$ & $\mathrm{C}$ & $2590.09 \mathrm{keV}^{28} \mathrm{Al}^{a}$ \\
\hline $2754.5 \pm 2.1^{*}$ & $\mathrm{Na}$ & A & \\
\hline $2821.5 \pm 1.2$ & ${ }^{28} \mathrm{Al}$ & $\mathrm{C}$ & $2821.29 \mathrm{keV}^{28} \mathrm{Al}^{a}$ \\
\hline $2959.8 \pm 1.9^{*}$ & $\mathrm{Mn},{ }^{28} \mathrm{Al}$ & $\mathrm{C}$ & $2960.01 \mathrm{keV}^{28} \mathrm{Al}^{a}$ \\
\hline $3033.3 \pm 1.6$ & ${ }^{28} \mathrm{Al}$ & $\mathrm{C}$ & $3033.748 \mathrm{keV}^{28} \mathrm{Al}^{a}$ \\
\hline $3413.0 \pm 1.8^{*}$ & ${ }^{57} \mathrm{Fe}$ & $\mathrm{C}$ & $3413.1 \mathrm{keV}^{57} \mathrm{Fe}^{a}$ \\
\hline $3436.7 \pm 1.4^{*}$ & ${ }^{57} \mathrm{Fe}$ & $\mathrm{C}$ & $3436.0 \mathrm{keV}^{57} \mathrm{Fe}^{a}$ \\
\hline $3464.6 \pm 1.4$ & ${ }^{28} \mathrm{Al}$ & $\mathrm{C}$ & $3464.95 \mathrm{keV}^{28} \mathrm{Al}^{a}$ \\
\hline $4133.3 \pm 1.5^{*}$ & ${ }^{28} \mathrm{Al}$ & $\mathrm{C}$ & $4133.1 \mathrm{keV}^{28} \mathrm{Al}^{a}$ \\
\hline $4218.3 \pm 2.4^{*}$ & ${ }^{57} \mathrm{Fe}$ & $\mathrm{C}$ & $4218.6 \mathrm{keV}^{57} \mathrm{Fe}^{a}$ \\
\hline $4259.2 \pm 1.7^{*}$ & ${ }^{28} \mathrm{Al}$ & $\mathrm{C}$ & $4259.46 \mathrm{keV}^{28} \mathrm{Al}^{a}$ \\
\hline $4690.6 \pm 1.9$ & ${ }^{28} \mathrm{Al}$ & $\mathrm{C}$ & $4690.60 \mathrm{keV}^{28} \mathrm{Al}^{a}$ \\
\hline $4733.7 \pm 1.6$ & ${ }^{28} \mathrm{Al}$ & $\mathrm{C}$ & $4733.85 \mathrm{keV}^{28} \mathrm{Al}^{a}$ \\
\hline $5409.2 \pm 2.5^{*}$ & ${ }^{28} \mathrm{Al}$ & $\mathrm{C}$ & $5410.6 \mathrm{keV}^{28} \mathrm{Al}^{a}$ \\
\hline $5920.2 \pm 1.8^{*}$ & ${ }^{57} \mathrm{Fe}$ & $\mathrm{C}$ & $5920.41 \mathrm{keV}^{57} \mathrm{Fe}^{a}$ \\
\hline
\end{tabular}

Table 3 (Contd.)

\begin{tabular}{llll}
\hline Energy $(\mathrm{keV})$ & Element & Process & Notes \\
\hline $\mathbf{6 0 1 8 . 4} \pm \mathbf{1 . 9}$ & ${ }^{57} \mathrm{Fe}$ & $\mathrm{C}$ & $6017.75 \mathrm{keV}^{57} \mathrm{Fe}^{a}$ \\
$\mathbf{6 1 1 0 . 7} \pm 5.0^{*}$ & ${ }^{36} \mathrm{Cl}$ & $\mathrm{C}$ & $6111.6 \mathrm{keV}^{36} \mathrm{Cl}^{a}$ \\
$\mathbf{6 7 6 8 . 2} \pm \mathbf{2 . 2}$ & ${ }^{7} \mathrm{Li}$ & $\mathrm{C}$ & $6769.4 \mathrm{keV}^{7} \mathrm{Li}^{a}$ \\
$\mathbf{7 1 2 0 . 4} \pm \mathbf{2 . 6}$ & ${ }^{57} \mathrm{Fe}$ & $\mathrm{C}$ & $7120.3 \mathrm{keV}^{57} \mathrm{Fe}^{a}$ \\
$\mathbf{7 1 3 4 . 5} \pm \mathbf{2 . 4}$ & ${ }^{57} \mathrm{Fe}$ & $\mathrm{C}$ & $7134.0 \mathrm{keV}^{57} \mathrm{Fe}^{a}$ \\
$\mathbf{7 2 1 2 . 9} \pm \mathbf{2 . 2}$ & ${ }^{28} \mathrm{Al}$ & $\mathrm{C}$ & $7212.8 \mathrm{keV}^{28} \mathrm{Al}^{a}$ \\
$\mathbf{7 2 7 8 . 9} \pm \mathbf{2 . 2}$ & ${ }^{57} \mathrm{Fe}$ & $\mathrm{C}$ & $7278.3 \mathrm{keV}^{57} \mathrm{Fe}^{a}$ \\
$\mathbf{7 6 3 1 . 5} \pm \mathbf{2 . 3}$ & ${ }^{57} \mathrm{Fe}$ & $\mathrm{C}$ & $7631.0 \mathrm{keV}^{57} \mathrm{Fe}^{a}$ \\
$\mathbf{7 6 4 5 . 7} \pm \mathbf{2 . 1}$ & ${ }^{57} \mathrm{Fe}$ & $\mathrm{C}$ & $7645.6 \mathrm{keV}^{57} \mathrm{Fe}^{a}$ \\
$\mathbf{7 7 2 4 . 3} \pm \mathbf{1 . 9}^{*}$ & ${ }^{28} \mathrm{Al}$ & $\mathrm{C}$ & $7723.85 \mathrm{keV}^{28} \mathrm{Al}^{a}$ \\
$\mathbf{8 8 8 6 . 0} \pm \mathbf{3 . 2}$ & ${ }^{*} \mathrm{Cr}$ & $\mathrm{C}$ & \\
$\mathbf{9 2 9 7 . 6} \pm \mathbf{3 . 0}$ & ${ }^{55} \mathrm{Fe}$ & $\mathrm{C}$ & $9295.8 \mathrm{keV}^{55} \mathrm{Fe}^{a}$ \\
$\mathbf{1 0 8 2 8 . 6} \pm \mathbf{4 . 4}$ & ${ }^{15} \mathrm{~N}$ & $\mathrm{C}$ & $10829.1 \mathrm{keV}^{15} \mathrm{~N}^{a}$
\end{tabular}

${ }^{a}$ T. Belgya and Z. Revay and G. L. Molnar. ${ }^{10} b$ G. Gilmore and J. D. Hemingway. ${ }^{23} c *$ Gamma-ray line on VESUVIO. ${ }^{d} \mathrm{~A}=$ activation, $\mathrm{AN}=$ annihilation, $\mathrm{C}=$ neutron capture, $\mathrm{IS}=$ inelastic scattering.

relative intensity of the gamma-ray line relative to its element. Gamma-ray lines of low intensity compared to the other lines of the same element (i.e. above 10) were not considered. All information collected on gamma-ray lines was integrated with those from ref. 10 .

\section{Results and discussion}

Tables 2 and 3 list the identified gamma-ray lines in the VESUVIO and INES beamline background. The tables contain the energy, element name, and creation process of the identified gamma-ray lines. The last column reports the energy and element of the gamma-ray lines measured in ref. 10 at the Budapest reactor and from a typical shielding in a counting room. ${ }^{23}$ Peaks of chemical elements identified from the drawings of the VESUVIO and INES beamlines are underlined. Peaks found on both beamlines are marked with a * symbol. The peak at $511 \mathrm{keV}$ is due to the electron-positron annihilation following pair production. The process can occur when gammas with energies above $1022 \mathrm{keV}$ are produced in neutron interactions. The $1885 \mathrm{keV}$ and $10831 \mathrm{keV}$ nitrogen lines are from the air and resins $\left(\mathrm{B}_{4} \mathrm{C}\right.$ binder) inside the blockhouse. The 2224 $\mathrm{keV}$ hydrogen line is from the blockhouse walls and the beam dump. The $478 \mathrm{keV}$ boron line is from walls, beam dump, and collimator. The aluminum lines are from the sample tank and collimator. The lithium peaks are from the neutron monitors and on VESUVIO from the detectors in backscattering. The iron lines are from the beam dump, flange, pipe, and collimator. The lead peaks are from the lead shielding and upstream collimation. The germanium peaks are from the activation of the HPGe detector. The gamma-ray lines at $749 \mathrm{keV}, 833 \mathrm{keV}$, and 8887 $\mathrm{keV}$ can be ascribed to chromium, and the $848 \mathrm{keV}, 1810 \mathrm{keV}$, $2112 \mathrm{keV}, 2959 \mathrm{keV}$ lines to manganese. Chromium and manganese are found in the composition of stainless steel grade $316 \mathrm{~L}$ used in flanges, pipes, and collimators. The $417 \mathrm{keV}$, $819 \mathrm{keV}, 1097 \mathrm{keV}, 1293 \mathrm{keV}, 1507 \mathrm{keV}, 1753 \mathrm{keV}$, and $2112 \mathrm{keV}$ 
lines could be assigned to indium, chemical element commonly used for metal seals. ${ }^{23}$ The $596 \mathrm{keV}, 610 \mathrm{keV}, 692 \mathrm{keV}, 848 \mathrm{keV}$, and $899 \mathrm{keV}$ lines are generated in inelastic scattering in germanium, iron, and lead. The $871 \mathrm{keV}$ line could be originated from the neutron capture in iron and oxygen.

The $1433 \mathrm{keV}$ and $123 \mathrm{keV}$ peaks are compatible with the activation and neutron capture of vanadium, a minor component of silver steel alloy used for collimators.

The $1434 \mathrm{keV}$ line is the most intense line of vanadium ${ }^{22}$ and the $125 \mathrm{keV}$ line is the second most intense. ${ }^{22}$ The third most intense line at $6517 \mathrm{keV}$ (ref. 22) is not visible; possible reasons are the low intensity ( $16 \%$ of the most intense line) $)^{22}$ and the low efficiency of the HPGe detector in this energy region. The 1367 $\mathrm{keV}$ and $2754 \mathrm{keV}$ peaks are compatible with the sodium activation in the collimator made of Borax. The most intense lines from sodium are $1368 \mathrm{keV}, 2754 \mathrm{keV}$, and $472 \mathrm{keV} .^{22}$ The third line at $472 \mathrm{keV}$ is not visible, probably buried in the $478 \mathrm{keV}$ boron peak. The $1172 \mathrm{keV}$ line can be assigned to the activation of cobalt in the iron structural materials. ${ }^{10}$ The $819 \mathrm{keV}, 972$ $\mathrm{keV}, 1172 \mathrm{keV}, 1293 \mathrm{keV}$, and $2113 \mathrm{keV}$ peaks could be ascribed to the radiative capture in tin, present in electronic soldering. The $559 \mathrm{keV}$ line is compatible with the strongest gammas from neutron capture in cadmium. The second and third Cd strongest lines at $651 \mathrm{keV}$ and $245 \mathrm{keV}$ are not visible, most likely due to the their low intensity (19\% and $14 \%$ of the strongest peak). Many peaks in the VESUVIO background spectrum were observed in ref. 10 at the Budapest reactor, and by Gilmore and Hemingway who reported a background spectrum in a typical shield in a counting room. ${ }^{23}$

Fig. 3 shows the gamma energy spectra on VESUVIO and INES. Both spectra were normalized to their integrated proton current and live time. A clear difference is visible in the Compton continuum shape. The gamma-ray background on INES shows a very large boron peak at $478 \mathrm{keV}$. The gamma-ray line is emitted by the decay of the excited ${ }^{7} \mathrm{Li}$ to the ground state generated in the reaction $n+{ }^{10} \mathrm{~B} \rightarrow{ }^{7} \mathrm{Li}^{*}+\alpha$. Peak to Background Ratios (PBR) of the $478 \mathrm{keV}$ boron peak are 1.8 on VESUVIO and 19.1 on INES, FWHM is $5.8 \mathrm{keV}$ on VESUVIO and 13.2 $\mathrm{keV}$ on INES. The Compton edge of the boron peak (310 $\mathrm{keV}$ ) is clearly visible in the INES spectrum, and not on
VESUVIO due to the smaller size of the peak. The annihilation peak is similar on both beamlines; the PBR is 4.3 on VESUVIO and 3.0 on INES.

The energy binning of $1.5 \mathrm{KeV}$ allowed us to observe differences in the boron peak line shapes from the VESUVIO and INES data (Fig. 4). These differences are due to the chemical matrix in which boron is embedded for the two beamlines. ${ }^{\mathbf{2 4 , 2 5}}$

INES displays a higher gamma-ray background in the energy ranges [180-511] $\mathrm{keV}$ and [2.4-7.6] $\mathrm{MeV}$, whereas in the region [511-2400] keV VESUVIO shows a higher background. Above 7.6 $\mathrm{MeV}$ the spectral shapes are similar. The differences can be attributed to the different blockhouses. In the pulse height spectra the region [180-530] keV contains 50.6\% (on VESUVIO) and $72.7 \%$ (on INES) of the total counts. Energy regions [5302000] $\mathrm{keV}$ and [2-7] MeV contain $39.7 \%$ and $8.9 \%$ on VESUVIO, and $17.0 \%$ and $9.5 \%$ on INES of the total counts. Gammas above $7 \mathrm{MeV}$ contribute only with $0.0009 \%$ on VESUVIO, and $0.0008 \%$ on INES.

Fig. 5 shows the VESUVIO gamma-ray background measured in this work compared with the spectrum reported in ref. 11 where the first characterization of the gamma background at the VESUVIO beamline was reported. The latter was normalized so to overlap the spectrum measured in this work in the energy region [100-150] keV. Main differences in the peak pattern are the presence of the $85 \mathrm{keV} \mathrm{X}$-ray $\mathrm{Pb}$ line, $570 \mathrm{keV}$ line inelastic

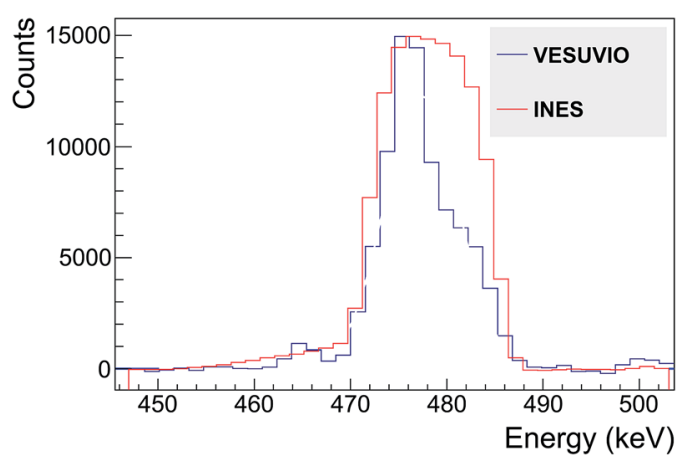

Fig. 4 Spectral shapes of the $478 \mathrm{keV}$ gamma-ray line normalized to the VESUVIO pulse height.
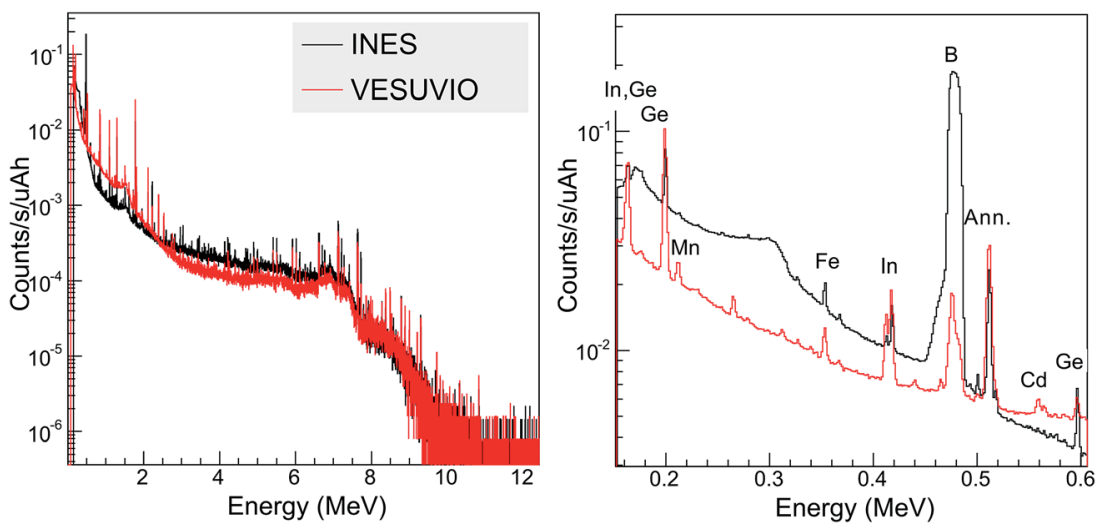

Fig. 3 Normalized gamma-ray background acquired on VESUVIO (red line) and INES (black line). Top: full range. Bottom: 150-600 keV region. 

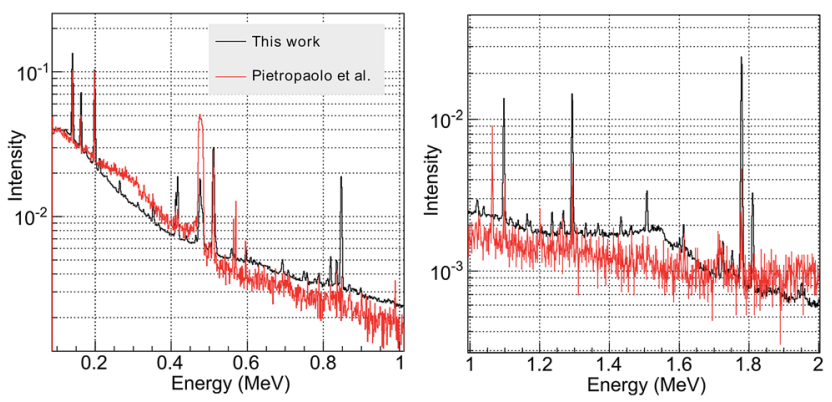

Fig. 5 VESUVIO gamma-ray background measured in this work (black line) and published in ref. 11 (red line). Left panel: energy range up to 1 MeV. Right panel: 1-2 MeV region.

scattering ${ }^{207} \mathrm{~Pb},{ }^{10,23} 564 \mathrm{keV}$ capture line $\mathrm{Sb}$ (usually found in lead), and $1064 \mathrm{keV}$ line inelastic scattering ${ }^{207} \mathrm{~Pb}^{23}$ in ref. 11. This is due to the lead shielding around the detector. Chlorine, oxygen, nitrogen, vanadium, chromium, and sodium peaks were identified in this work only.

Differences in spectra and peak intensities between the present measurement and those reported in ref. 11 reflect the changes and upgrades on the VESUVIO beamlines occurred between 2008 and 2011. These differences reflect the changes and upgrades on the VESUVIO beamlines occurred between 2008 and 2011. To the best of our knowledge no changes in the shielding configuration have been made since 2006. We therefore ascribe the differences shown in Fig. 5 to the modification of the inelastic neutron scattering components: in particular this regarded the installation of new scattering detector modules and neutron energy analyzer set-ups and the associated electronic devices. ${ }^{4}$

Germanium, indium, iron, boron, aluminum, and manganese lines were present in both spectra.

\section{Conclusions}

The gamma-ray background of the VESUVIO and INES beamlines at the spallation neutron source ISIS was measured with a HPGe spectrometer. Sources and creation processes of more than 60 gamma-ray lines in the energy range $80 \mathrm{keV}-12 \mathrm{MeV}$ were identified by using specifically developed data analysis software and were correlated with components of the beamlines. Comparison of gamma-ray backgrounds of the two beamlines shows that the INES background was dominated by low-energy photons while the VESUVIO background by highenergy photons. The reason could be the different neutron shielding on INES ${ }^{\mathbf{2 6}}$ and VESUVIO beamlines. The boron peak shape shows differences that could be attributed to Doppler broadening due to the different chemical environment where boron nucleus is produced by neutron capture. Many of the gamma-ray lines found in this work were present also in the Budapest reactor gamma-ray background measured by Belgya and co-workers, with the main exception of gamma lines from elements in steel and aluminium alloys. Next generation irradiation beamlines, like for instance CHIPIR at ISIS target station 2, have different shielding materials with respect to conventional neutron scattering spectrometers; this is expected to result in a different background, which will have to be investigated with dedicated measurements. This work provides insight into the processes which cause gamma-ray background, aiming at providing information for the development of new strategies for gamma-ray background reduction in existing and future beamlines.

\section{Acknowledgements}

This work was supported by the CNR-STFC Agreement no. 06/ 20018 concerning collaboration in scientific research at the spallation neutron source ISIS within the PANAREA project.

\section{References}

1 C. Andreani, A. D'Angelo, G. Gorini, S. Imberti, A. Pietropaolo, N. J. Rhodes, E. M. Schooneveld, R. Senesi and M. Tardocchi, CdZnTe $\gamma$ detector for deep inelastic neutron scattering on the VESUVIO spectrometer, Appl. Phys. A: Mater. Sci. Process., 2004, 78, 903.

2 H. Harada, S. Goto, et al. Study of Neutron Capture Reactions Using the $4 \pi$ Ge Spectrometer, J. Korean Phys. Soc., 2011, 59, 1585-1588.

3 R. Bedogni, A. Esposito, C. Andreani, R. Senesi, M. P. De Pascale, P. Picozza, A. Pietropaolo, G. Gorini, C. D. Frost and S. Ansell, Characterization of the neutron field at the ISIS-VESUVIO facility by means of a bonner sphere spectrometer, Nucl. Instrum. Meth. Phys. Res. A, 2009, 612(1), 143-148.

4 J. Mayers and G. F. Reiter, The VESUVIO electron volt neutron spectrometer, Meas. Sci. Technol., 2012, 23, 045902.

$5 \mathrm{~J}$. Hori, et al. Measurement of Neutron Capture Gamma Rays from the Resonances of and $96 \mathrm{Zr}$ at the J-PARC/MLF/ANNRI 91 Zr, J. Korean Phys. Soc., 2011, 59, 1777-1780.

6 K. Ryzewski, S. Herringer, H. Bilheux, L. Walker, B. Sheldon, S. Voisin, J. C. Bilheux and V. Finocchiaro, Neutron Imaging of Archaeological Bronzes at the Oak Ridge National Laboratory, Phys. Procedia, 2013, 43, 343-351.

7 A. Pietropaolo and R. Senesi, Electron Volt neutron spectrometers, Phys. Rep., 2011, 508, 45-90.

8 R. Nunez-Lagos and A. Virto, Shielding and background reduction, Appl. Radiat. Isot., 1996, 47, 1011.

9 Y. Kasugai, M. Ooi and T. Kai, Gamma dose measurements and spectroscopy analysis for spallation products in JSNS mercury circulation system, Prog. Nucl. Sci. Technol., 2011, 1, 501-504.

10 T. Belgya, Z. Revay and G. L. Molnar, Gamma ray background at the Budapest PGAA facility, J. Radioanal. Nucl. Chem., 2005, 265, 181.

11 A. Pietropaolo, M. Tardocchi, E. M. Schooneveld and R. Senesi, Characterization of the gamma background in epithermal neutron scattering measurements at pulsed neutron sources, Nucl. Instrum. Meth. Phys. Res. A, 2006, 568, 826-838.

12 http://www.isis.stfc.ac.uk/instruments/vesuvio/vesuvio4837. html. 
13 C. Andreani, D. Colognesi, J. Mayers, G. F. Reiter and R. Senesi, Measurement of momentum distribution of light atoms and molecules in condensed matter systems using inelastic neutron scattering, Adv. Phys., 2005, 54, 377.

14 http://www.isis.stfc.ac.uk/instruments/ines/ines7450.html.

15 ORTEC. http://www.ortec-online.com. 2014.

16 R. Brun and F. Rademakers, Root - an object oriented data analysis framework, Proceedings AIHENP'96 Workshop, Lausanne, 1996, http://root.cern.ch/.

17 R. Brun and F. Rademakers, Root - an object oriented data analysis framework, Nucl. Instrum. Meth. Phys. Res. A, 1997, 389, 81.

18 M. Morhac, J. Kliman, V. Matousek, M. Veselsky and I. Turzo, Background elimination methods for multidimensional coincidence gamma-ray spectra, Nucl. Instrum. Meth. Phys. Res. A, 1997, 401, 113-132.

19 M. Morhac, J. Kliman, V. Matousek, M. Veselsky and I. Turzo, Efficient one- and two-dimensional Gold deconvolution and its application to gamma-ray spectra decomposition, Nucl. Instrum. Meth. Phys. Res. A, 1997, 401, 385-408.
20 M. Morhac, J. Kliman, V. Matousek, M. Veselský and I. Turzo, Identification of peaks in multidimensional coincidence gamma-ray spectra, Nucl. Instrum. Meth. Phys. Res. A, 2000, 443, 108-125.

21 G. L. Molnar, Handbook of Prompt Gamma Activation Analysiswith neutron beams, Kluwer Academic Publishers, 2004.

22 IAEA, International Atomic Energy Agency, Database for Prompt Gamma-ray Neutron Activation Analysis, 2013.

23 G. Gilmore and J. D. Hemingway, Practical Gamma-Ray Spectrometry, Wiley, Chichester, 1995.

24 L. Szentmiklosi, K. Gmeling and Z. Revay, Fitting the boron peak and resolving interferences in the $450 \mathrm{keV}$ region of PGAA spectra, J. Radioanal. Nucl. Chem., 2007, 271, 447-453.

25 Y. Sakai, M. K. Kubo, H. Matsue and C. Yonezawa, Biological application of Doppler broadening of neutron-inducted prompt gamma-ray from energetic 7Li, J. Radioanal. Nucl. Chem., 2005, 265, 287-290.

26 M. Celli, F. Grazzi, M. Zoppi. A new ceramic material for shielding pulsed neutron scattering instruments. Nucl. Instrum. Meth. Phys. Res. A, 565-2, 2006. 